\title{
Capsule Endoscopy in Refractory Diarrhea-Predominant Irritable Bowel Syndrome and Functional Abdominal Pain
}

\author{
Manuel Valero, Gladys Bravo-Velez, Roberto Oleas, Miguel Puga-Tejada, Miguel Soria-Alcívar, Haydee Alvarado Escobar, \\ Jorge Baquerizo-Burgos, Hannah Pitanga-Lukashok and Carlos Robles-Medranda \\ Gastroenterology and Endoscopy Division, Instituto Ecuatoriano de Enfermedades Digestivas (IECED), Guayaquil, Ecuador
}

Background/Aims: Capsule endoscopy is a diagnostic method for evaluating the small bowel lumen and can detect undiagnosed lesions. The aim of this study was to evaluate the diagnostic yield and clinical impact of capsule endoscopy in patients with refractory diarrhea-predominant irritable bowel syndrome and functional abdominal pain.

Methods: This study involved a retrospective analysis of prospectively collected data, maintained in a database. Patients with refractory diarrhea-predominant irritable bowel syndrome and functional abdominal pain within the period of March 2012 to March 2014 were included. Capsule endoscopy was used to detect small bowel pathologies in both groups.

Results: Sixty-five patients (53.8\% female) fulfilled the inclusion criteria and had a mean ( \pm standard deviation) age of $50.9 \pm 15.9$ years. Clinically significant lesions were detected via capsule endoscopy in $32.5 \%$ of the patients in the abdominal pain group and $54.5 \%$ of the patients in the diarrhea group. Overall, $48 \%$ of patients had small bowel pathologies detected during the capsule endoscopy study. Inflammatory lesions and villous atrophy were the most frequent lesions identified in $16.9 \%$ and $15.3 \%$ of patients in the abdominal pain and the diarrhea groups, respectively.

Conclusions: Routine use of capsule endoscopy in patients with irritable bowel syndrome should not be recommended. However, in patients with refractory conditions, capsule endoscopy may identify abnormalities. Clin Endosc 2018;51:570-575

Key Words: Capsule endoscopy; Irritable bowel syndrome; Refractory

\section{INTRODUCTION}

Irritable bowel syndrome (IBS) is one of the most common gastrointestinal disorders. Its prevalence in the general population is approximately $5 \%-20 \%$, and the condition is more common in women and young adults. ${ }^{1}$ Despite IBS being one of the most frequent reasons for consultation, many patients are misdiagnosed owing to a lack of reliable biomarkers or en-

Received: March 14, 2018 Revised: June 9, 2018

Accepted: July 3, 2018

Correspondence: Carlos Robles-Medranda

Gastroenterology and Endoscopy Division, Instituto Ecuatoriano de Enfermedades Digestivas (IECED), OMNI Hospital, Av. Abel Romeo Castillo y Av. Juan

Tanca Marengo, Torre Vitalis, Mezzanine 3, Guayaquil 090505, Ecuador

Tel: +593-4-2109180, Fax: +593-4-2109180, E-mail: carlosoakm@yahoo.es ORCID: https://orcid.org/0000-0003-2434-3369

(c) This is an Open Access article distributed under the terms of the Creative Commons Attribution Non-Commercial License (http://creativecommons.org/ licenses/by-nc/3.0) which permits unrestricted non-commercial use, distribution, and reproduction in any medium, provided the original work is properly cited. doscopic findings. Endoscopic evaluation, which is performed to exclude other etiologies, is normal in more than $50 \%$ of cases. Further evaluation is not recommended for gastrointestinal functional disorders, and clinical diagnosis based on the Rome IV criteria is necessary in such cases. ${ }^{2}$

IBS is characterized by chronic or recurrent abdominal pain associated with changes in bowel frequency and consistency. Functional abdominal pain is not associated with physiological events and cannot be fully explained by other medical conditions. Previous studies have demonstrated that the presence of Rome IV criteria, in the absence of red flags, exhibit a sensitivity and specificity of $62.7 \%$ and $97.1 \%$, respectively. ${ }^{3}$ However, Rome IV criteria can be present in other organic or functional gastrointestinal disorders, and it is possible that patients could be misdiagnosed as having IBS, especially when the presenting symptom is unresponsive to treatment.

Capsule endoscopy (CE) is a diagnostic method that enables visualization of the small bowel lumen and can diagnose 
lesions not previously detected by other methods. ${ }^{4}$ Several studies have demonstrated that $\mathrm{CE}$ is superior to barium follow-through, push enteroscopy, and computed tomography for the diagnosis of small bowel disease..$^{5-7}$

The aim of this study was to evaluate the diagnostic yield and clinical impact of $\mathrm{CE}$ in patients with refractory diarrhea-predominant IBS (D-IBS) and functional abdominal pain.

\section{MATERIALS AND METHODS}

\section{Study design}

A retrospective analysis of prospectively collected data, maintained in a database, was performed with a review of consecutive medical records and CEs. Referred patients were enrolled at the Instituto Ecuatoriano de Enfermedades Digestivas (IECED) for CE studies from March 2012 to March 2014.

The study protocol was approved by the Institutional Review Board (Number: 12-05-2015). The study was conducted according to the Declaration of Helsinki.

\section{Population selection}

Patients older than 18 years with D-IBS and functional abdominal pain with no improvement after medical treatment were included in separate groups. Medical records, imaging, and laboratory tests were reviewed by two gastroenterologists for the exclusion of potential misdiagnoses.

The diagnosis of D-IBS and functional abdominal pain was based on Rome III criteria. Stool consistencies using the Bristol Stool Form Score (BSFS), between 6-7 in greater than 25\% of bowel movements, were considered as diarrhea.

No medical improvement was considered when the main symptom (diarrhea or abdominal pain) did not improve after eight weeks of medical treatment with $40 \mathrm{mg}$ otilonium bromide three times daily and $200 \mathrm{mg}$ trimebutine twice daily for abdominal pain, or 4-8 $\mathrm{mg}$ loperamide hydrochloride daily and $200 \mathrm{mg}$ rifaximin three times daily for three days for diarrhea, as treatment modalities. Medical improvement was defined as the absence of pain and a BSFS of 3-5. Clinical impact was defined as the change in medical management resulting from $\mathrm{CE}$ study results.

Patients with incomplete medical charts were excluded. Any clinical, laboratory, or imaging evidence of suspected organic disease was reviewed for potential misdiagnosis and exclusion from the analysis. Patients with abnormal ultrasonography and computed tomography images and elevated inflammatory biomarkers (erythrocyte sedimentation rate and/or C-reactive protein) were excluded. Fecal calprotectin levels were measured and recorded to rule out Crohn's disease. Patients with celiac disease (based on IgA tissue transglutaminase antibodies), lactose intolerance and bacterial overgrowth (based on a breath test), parasitic infections (based on a stool examinations), and patients on nonsteroidal anti-inflammatory drugs were all excluded. Any abnormal findings on upper endoscopy and/or colonoscopy were also criteria for exclusion (Fig. 1).

After selection, patients were allocated into two groups based on their main presenting symptoms. Group A included patients with D-IBS, whereas group B included patients with functional abdominal pain. The main symptom was selected according to the symptom that led patients to seek consultation. Constipation-predominant IBS patients were excluded due to ethical concerns about capsule retention, and CE is currently not indicated in these cases.

A gastroenterologist/CE expert, blind to medical charts, reviewed all capsule images.

\section{Capsule endoscopy technique}

Two types of capsules were used: MiroCam (Intromedic, Seoul, Korea) and Given PillCam SB2 Diagnostic System (Given Imaging Ltd., Yoqneam, Israel). Patients maintained a liquid diet one day prior to the study. An eight-hour fasting period before capsule intake was mandatory in all cases. Oral intake of $25 \mathrm{mg}$ levosulpiride two hours before the procedure was part of the institutional protocol. When the capsule was

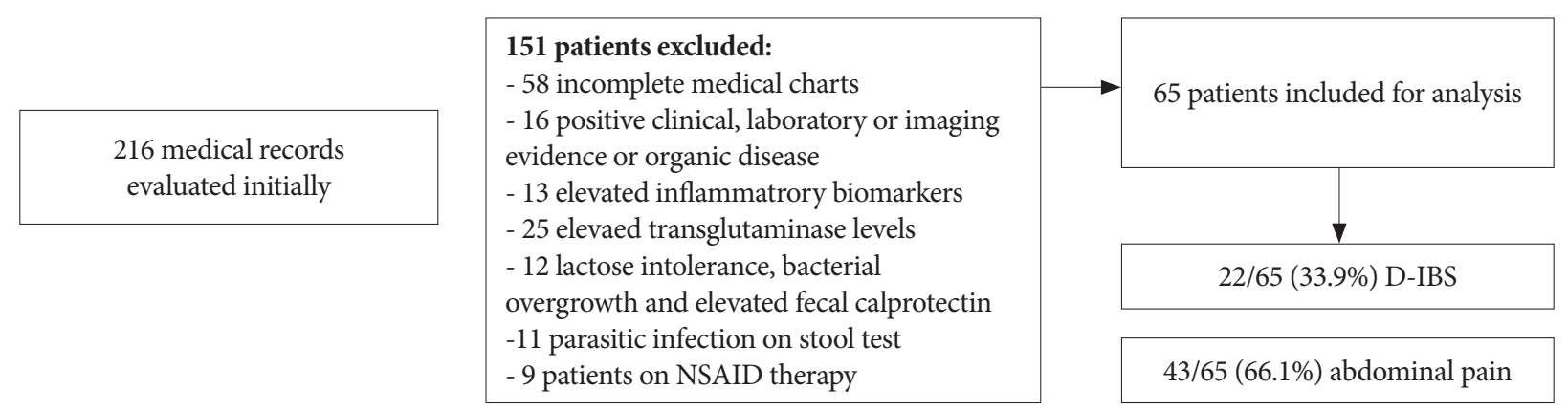

Fig. 1. Flowchart describing the process of population selection. NSAID, nonsteroidal anti-inflammatory agent; D-IBS, diarrhea-predominant irritable bowel syndrome. 
activated, the patient ingested it with $250 \mathrm{cc}$ of water. The CE procedure lasted approximately eight hours.

\section{Statistical analysis}

Continuous variables were analyzed according to their statistical distribution calculated by Shapiro-Wilk or Kolmogorov-Smirnov tests as indicated. Normally distributed data are represented as means (standard deviation), and skewed distributed data are represented as medians (interquartile range). Qualitative variables are represented as frequencies (\%). Demographics and CE findings were compared between groups using Student's or Welch's $t$-tests for continuous variables and Pearson's chi-square or Fisher's exact test for categorical variables. A $p$-value $<0.05$ was considered statistically significant. Data analysis was assessed by the IECED institutional biostatistician. All statistical analyses were performed using R v3.4.3 (R Foundation for Statistical Computing, Vienna, Austria).

\section{RESULTS}

A total of 216 medical records were evaluated by two gastroenterologists for inclusion and exclusion fulfillment criteria. Sixty-five patients (30\%) were included for analysis. Another gastroenterologist, blind to the medical records and also an expert in CE, reviewed the images. The mean age was 50.2 years, and $35(53.8 \%)$ participants were female. The main presenting symptom was diarrhea in 22 patients (33.9\%) and abdominal pain in 43 patients $(66.1 \%)$. The mean level of fecal calprotectin in the abdominal pain group was $18.2 \pm 6.4$ and 25.3 \pm 9.2 for the D-IBS group. Demographic characteristics in both groups are summarized in Table 1 .

In 28 patients (43\%), findings were detected during CE. Clinically significant small bowel pathologies were identified in 26 patients $(40 \%)(p$-value $=0.117)$, with a clinical impact of $92.8 \%$. Inflammatory lesions and villous atrophy were the most frequent lesions found in $16.9 \%$ and $15.3 \%$ of patients, respectively.

Clinically significant lesions detected by $\mathrm{CE}$ were noted in 12 patients $(54.6 \%)$ in group A $(n=22)$. Villous atrophy and Crohn's disease-related ulcers were observed in 4 (18.2\%) and $8(36.4 \%)$ patients, respectively. Normal study findings were noted in 10 patients $(45.4 \%)$.

Clinically significant lesions were detected in 14 patients $(32.5 \%)$ in group B $(n=43)$. Non-specific erythema was detected in 2 patients (4.7\%); however, these cases were not considered clinically relevant. Ascariasis was identified in 1 patient (2.3\%) (Fig. 2A). Ulcers detected during CE (Fig. 2B) were biopsied in follow-up enteroscopy, and Crohn's disease was diagnosed in 11 cases (100\%). Villous atrophy confirmed by biopsy supported the diagnosis of celiac disease in 10 cases (100\%). The submucosal tumor detected by CE was a gastrointestinal stromal tumor confirmed by histopathology (Fig. 2C). Villous atrophy was detected by CE in the jejunum (Fig. 2D). Three gastroparesis cases (6.9\%) were suspected due to a prolonged time for CE passage through the small bowel and the presence of food despite the fasting protocol before CE intake and prokinetics administration. These cases were confirmed by scintigraphy. The distribution of findings detected during CE are described in Table 2.

\section{DISCUSSION}

Patients with D-IBS and functional abdominal pain diagnosis might be challenging, especially in patients with no response to medical treatment. This study evaluated the diagnostic yield and clinical impact of CE for detecting small bowel pathologies among these groups of patients.

Current diagnostic methods fail to obtain an adequate diagnosis. No specific findings discriminate IBS from healthy control patients. ${ }^{1}$ Two trials reported a $10 \%-14 \%$ incidence of mucosal breaks observed via $\mathrm{CE}$ in healthy controls, whereas another two trials detected normal mucosa in control

Table 1. Demographic Characteristics

\begin{tabular}{lccc}
\hline & $\begin{array}{c}\text { Group A } \\
\text { (D-IBS) }\end{array}$ & $\begin{array}{c}\text { Group B } \\
\text { (Functional abdominal pain) }\end{array}$ & Total \\
\hline No. of patients (\%) & $22(33.9 \%)$ & $43(66.1 \%)$ & 65 \\
Sex, $n(\%)$ & & & $35(53.8 \%)$ \\
Female & $9(41 \%)$ & $26(60 \%)$ & $30(46.2 \%)$ \\
Male & $13(59 \%)$ & $17(40 \%)$ & $50.9(15.9)$ \\
Mean age, yr (SD) & $53.3(16.9)$ & $49.6(15.5)$ & 21.7 \\
Fecal calprotectin levels $(\mathrm{mg} / \mathrm{kg}$ of stool) & 25.3 & 18.2 & \\
\hline
\end{tabular}

D-IBS, diarrhea-predominant irritable bowel syndrome; SD, standard deviation. 

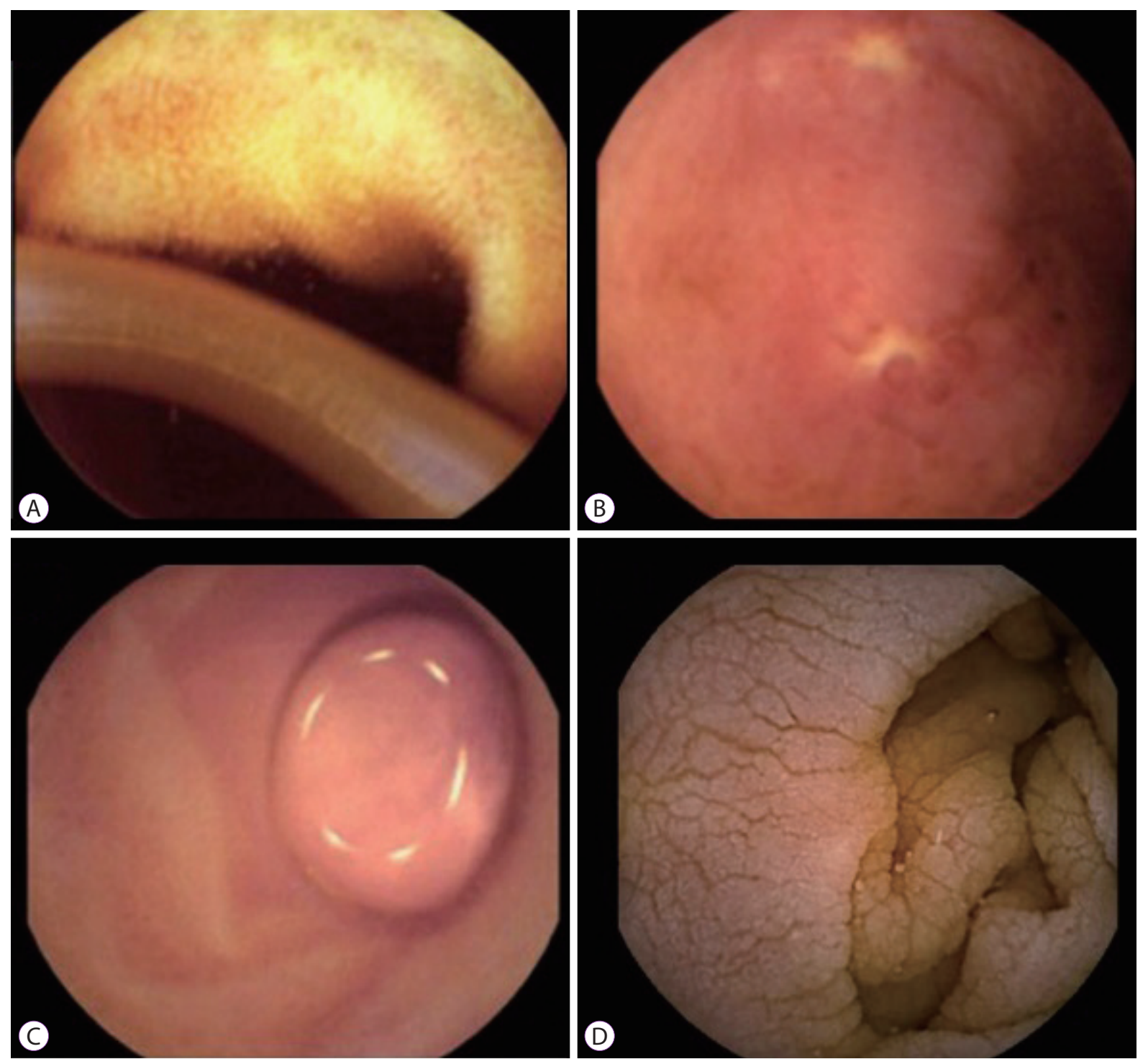

Fig. 2. Capsule endoscopy (CE) findings. (A) Ascariasis found in the small intestine in a patient with persistent abdominal pain, (B) ulcer identified by CE in the terminal ileum. Crohn's disease was confirmed by histopathology of an enteroscopic sample, (C) sub-mucosal tumor in the upper jejunum, (D) villous atrophy detected by $C E$ in jejunum.

Table 2. Lesions Detected via Capsule Endoscopy

\begin{tabular}{|c|c|c|c|c|c|c|}
\hline & & p A (D & & Group & tional a & minal pain) \\
\hline & Female & Male & Total, $n / 22(\%)$ & Female & Male & Total, $n / 43(\%)$ \\
\hline Villous atrophy & 2 & 2 & $4 / 22(18.2 \%)$ & 4 & 2 & $6 / 43(13.9 \%)$ \\
\hline Crohn's Disease related ulcer & 1 & 7 & $8 / 22(36.4 \%)$ & 1 & 2 & $3 / 43(6.9 \%)$ \\
\hline Ascariasis & & & & 1 & - & $1 / 43(2.3 \%)$ \\
\hline Gastroparesis & & & & 1 & 2 & $3 / 43(6.9 \%)$ \\
\hline Submucosal Tumor & & & & - & 1 & $1 / 43(2.3 \%)$ \\
\hline Erythema & & & & 1 & 1 & $2 / 43(4.6 \%)$ \\
\hline Normal mucosa & 6 & 4 & $10 / 22(45.4 \%)$ & 18 & 9 & $27 / 43(62.8 \%)$ \\
\hline Clinical impact & & & $12 / 22(54.6 \%)$ & & & $14 / 43(32.5 \%)$ \\
\hline
\end{tabular}

D-IBS, diarrhea-predominant irritable bowel syndrome. 
patients. ${ }^{8-11}$ On the other hand, there are some suggestions that organic gastrointestinal disorders (inflammatory bowel disease, colorectal cancer, or infectious diarrhea) can be identified in subsets of patients who fulfill Rome IV criteria for IBS. $^{12}$

Although IBS diagnoses were based on Rome III criteria in this study due to the time frame of patients' enrollment, currently available Rome IV criteria had a comparable sensitivity and specificity to that reported for Rome III. ${ }^{3}$

CE has become an important method in the diagnosis of small bowel pathologies, ${ }^{13}$ including occult gastrointestinal bleeding, Crohn's disease, celiac disease, inherited polyposis syndromes, and small bowel tumors. ${ }^{13-18}$

Chronic diarrhea and abdominal pain are indications for CE. Current data suggest that CE should not be the first-line diagnostic test. ${ }^{19,20}$ Song et al., demonstrated the positive diagnostic yield of CE (42.9\%) in patients with chronic diarrhea, in whom the final diagnosis changed in $31.4 \%$ of patients, with Crohn's disease being the most common finding. ${ }^{21}$ In a systematic review of $\mathrm{CE}$ and unexplained chronic abdominal pain, a limited diagnostic yield of $20.9 \%$ was described. Inflammatory lesions were the most common bowel pathologies identified by CE (78.3\%); however, increased heterogeneity between studies was found. ${ }^{22}$

CE should be used when an organic disease is suspected or in the presence of alarm features. In patients with abdominal pain associated with weight loss, fever, anemia, positive stool guaiac test, or signs of inflammation, the possibility of detecting findings on CE increases from $18 \%$ to $41.7 \%^{22,23}$

Ohlsson et al., demonstrated that in $26 \%$ of patients with IBS-small bowel pathologies, findings can be observed when using CE. ${ }^{12}$ However, they did not identify macroscopic abnormalities that could explain the symptoms in the majority of patients with IBS and concluded that routine use of CE in patients with IBS should not be recommended. Furthermore, they considered that a subgroup of patients initially classified with IBS with persistently severe or aggravating symptoms should undergo further investigation with $\mathrm{CE} .{ }^{12}$ Kalla et al., evaluated patients with D-IBS using CE and identified evidence of subtle mucosal changes in $30 \%$ of patients. ${ }^{24}$

The present study revealed that $54.5 \%$ and $32.5 \%$ of cases unresponsive to medical treatment in groups $\mathrm{A}$ and $\mathrm{B}$, respectively, exhibited clinically relevant pathological findings on CE.

In the present cohort, $\mathrm{CE}$ had an important clinical impact in 26 cases (40\%), changing the clinical management of these patients.

There were some limitations in the present study. Namely, it was a retrospective, non-randomized, single-center study with no controls. Additionally, all CE images were evaluated by one expert.

In conclusion, CE had a favorable diagnostic yield and clinical impact on the management of patients with D-IBS and functional abdominal pain unresponsive to medical treatment. Routine use of CE in IBS patients should not be recommended. However, in patients with a lack of response to appropriate medical treatment, CE may identify abnormalities, thereby improving medical management.

\section{Conflicts of Interest}

The authors have no financial conflicts of interest.

\section{Author Contributions}

Conceptualization: Carlos Robles-Medranda, Hannah Pitanga-Lukashok Data curation: Manuel Valero, Gladys Bravo-Velez, Miguel Puga-Tejada Formal analysis: MPT, Roberto Oleas, Jorge Baquerizo-Burgos

Investigation: HPL, MV, GBV, Miguel Soria-Alcívar, Haydee Alvarado Escobar

Methodology: CRM, HPL, MPT, RO, JBB

Project administration: CRM, HPL, GBV, MV

Supervision: CRM, HPL

Validation: MPT

Visualization: CRM, HPL, GBV, RO, JBB

Writing-original draft: CRM, HPL, MV, GBV

Writing-review \& editing: MV,GBV, RO, MPT, MSA, HAE, JBB, HPL, CRM

\section{REFERENCES}

1. Longstreth GF, Thompson WG, Chey WD, Houghton LA, Mearin F, Spiller RC. Functional bowel disorders. Gastroenterology 2006;130:14801491.

2. Mearin F, Lacy BE, Chang L, et al. Bowel disorders. Gastroenterology 2016 Feb 18 [Epub]. https://doi.org/10.1053/j.gastro.2016.02.031.

3. Palsson OS, Whitehead WE, van Tilburg MAL, et al. Development and validation of the Rome IV diagnostic questionnaire for adults. Gastroenterology 2016;150:1481-1491.

4. Yang L, Chen Y, Zhang B, et al. Increased diagnostic yield of capsule endoscopy in patients with chronic abdominal pain. PLoS One 2014;9:e87396.

5. Appleyard M, Fireman Z, Glukhovsky A, et al. A randomized trial comparing wireless capsule endoscopy with push enteroscopy for the detection of small-bowel lesions. Gastroenterology 2000;119:1431-1438.

6. Costamagna G, Shah SK, Riccioni ME, et al. A prospective trial comparing small bowel radiographs and video capsule endoscopy for suspected small bowel disease. Gastroenterology 2002;123:999-1005.

7. Hara AK, Leighton JA, Sharma VK, Fleischer DE. Small bowel: preliminary comparison of capsule endoscopy with barium study and CT. Radiology 2004;230:260-265.

8. Goldstein JL, Eisen GM, Lewis B, Gralnek IM, Zlotnick S, Fort JG. Video capsule endoscopy to prospectively assess small bowel injury with celecoxib, naproxen plus omeprazole, and placebo. Clin Gastroenterol Hepatol 2005;3:133-141.

9. Graham DY, Opekun AR, Willingham FF, Qureshi WA. Visible small-intestinal mucosal injury in chronic NSAID users. Clin Gastroenterol Hepatol 2005;3:55-59.

10. Maiden L, Thjodleifsson B, Theodors A, Gonzalez J, Bjarnason I. A quantitative analysis of NSAID-induced small bowel pathology by cap- 
sule enteroscopy. Gastroenterology 2005;128:1172-1178.

11. Adler SN, Jacob H. Occult inflammatory small-bowel disease: not so occult anymore. Scand J Gastroenterol 2005;40:360-364.

12. Ohlsson B, Bengtsson M, Nielsen J, Toth E. A prospective evaluation of the diagnostic value of video capsule endoscopy in patients initially classified as irritable bowel syndrome. Eur J Intern Med 2009;20:48-52.

13. Pennazio M, Spada C, Eliakim R, et al. Small-bowel capsule endoscopy and device-assisted enteroscopy for diagnosis and treatment of small-bowel disorders: European Society of Gastrointestinal Endoscopy (ESGE) clinical guideline. Endoscopy 2015;47:352-376.

14. Cobrin GM, Pittman RH, Lewis BS. Increased diagnostic yield of small bowel tumors with capsule endoscopy. Cancer 2006;107:22-27.

15. Mata A, Llach J, Castells A, et al. A prospective trial comparing wireless capsule endoscopy and barium contrast series for small-bowel surveillance in hereditary GI polyposis syndromes. Gastrointest Endosc 2005;61:721-725.

16. Culliford A, Daly J, Diamond B, Rubin M, Green PH. The value of wireless capsule endoscopy in patients with complicated celiac disease. Gastrointest Endosc 2005;62:55-61.

17. Fireman Z, Mahajna E, Broide E, et al. Diagnosing small bowel Crohn's disease with wireless capsule endoscopy. Gut 2003;52:390-392.
18. Nakamura M, Niwa Y, Ohmiya N, et al. Preliminary comparison of capsule endoscopy and double-balloon enteroscopy in patients with suspected small-bowel bleeding. Endoscopy 2006;38:59-66.

19. Spada C, Pirozzi GA, Riccioni ME, Iacopini F, Marchese M, Costamagna G. Capsule endoscopy in patients with chronic abdominal pain. Dig Liver Dis 2006;38:696-698.

20. Fry LC, Carey EJ, Shiff AD, et al. The yield of capsule endoscopy in patients with abdominal pain or diarrhea. Endoscopy 2006;38:498-502.

21. Song HJ, Moon JS, Jeon SR, et al. Diagnostic yield and clinical impact of video capsule endoscopy in patients with chronic diarrhea: a Korean multicenter CAPENTRY study. Gut Liver 2017;11:253-260.

22. Xue M, Chen X, Shi L, Si J, Wang L, Chen S. Small-bowel capsule endoscopy in patients with unexplained chronic abdominal pain: a systematic review. Gastrointest Endosc 2015;81:186-193.

23. May A, Manner H, Schneider M, Ipsen A, Ell C. Prospective multicenter trial of capsule endoscopy in patients with chronic abdominal pain, diarrhea and other signs and symptoms (CEDAP-Plus Study). Endoscopy 2007;39:606-612.

24. Kalla R, McAlindon ME, Sanders DS, Sidhu R. Subtle mucosal changes at capsule endoscopy in diarrhoea predominant irritable bowel syndrome. Med Hypotheses 2012;79:423. 